\section{Classification of Two-Phase Flow Patterns by Ultrasonic Sensing}

\author{
Devesh K. Jha \\ e-mail: dkj5042@psu.edu
}

\author{
Asok Ray \\ Fellow ASME \\ e-mail: axr2@psu.edu
}

Kushal Mukherjee
Mem. ASME
e-mail: kum162@psu.edu

Department of Mechanical Engineering,

Pennsylvania State University,

University Park, PA 16802

\author{
Subhadeep Chakraborty \\ Department of Mechanical Engineering, \\ University of Tennessee, \\ Knoxville, TN 37996 \\ e-mail: schakrab@utk.edu
}

This paper presents a methodology for classification of twophase flow patterns in fluid systems, which takes the measurements of an in situ ultrasonic sensor as inputs. In contrast to the common practice of having an array of ultrasonic detectors, the underlying algorithm requires only a single sensor hardware in combination with an integrated software of signal conditioning, feature extraction, and pattern classification. The proposed method is noninvasive and can be implemented in a variety of industrial applications (e.g., petrochemical processes and nuclear power plants). This concept of flow pattern classification is experimentally validated on a laboratory test apparatus. [DOI: 10.1115/1.4007555]

Keywords: pattern classification, ultrasonic measurements, twophase flow, symbolic dynamic analysis

\section{Introduction}

In a two-phase flow system, the distribution of vapor and liquid parts follows a variety of patterns, where the diversity in flow patterns may take place due to relative velocities at the interface between the phases in addition to several other factors such as the bulk flow rate, fluid properties, and flow boundary conditions [1]. Characterization of flow patterns and identification of the associated flow regimes are critical for design, analysis, and operation in a variety of industries such as petrochemical processes and nuclear steam power plants.

Two-phase flow patterns are characterized based on the extent of their departure from the single-phase liquid as bubbly, capillary (also called cap-bubbly), slug, churn, annular, and the singlephase vapor, respectively. Three commonly occurring two-phase flow patterns, namely, bubbly, cap-bubbly, and slug, as shown in Fig. 1, are investigated in this paper.

Contributed by the Dynamic Systems Division of ASME for publication in the Journal of Dynamic Systems, Measurement, and Control. Manuscript received December 24, 2011; final manuscript received July 18, 2012; published online November 7, 2012. Assoc. Editor: Douglas Adams.
A significant amount of research has been accomplished for prediction of void fraction in two-phase flow systems. However, comparatively much less work has been reported in open literature for classification of different flow patterns in two-phase flow systems, and some of these reported methods require a large array of sensors and associated instrumentation, both for void fraction identification and flow pattern classification. High-speed photography, X-ray, and similar imaging techniques allow flow patterns to be discovered from direct observation. Although these methods are not very expensive and, in most cases, are not difficult to execute, their procedures and derived inferences are somewhat subjective. The major difficulty in pattern classification solely based on direct observations, even using high-speed photography, is that the picture is often ambiguous and is therefore difficult to interpret, specially when dealing with high flow velocities. Among the currently available techniques, the computed tomography (CT) method has gained prominence. For example, Bieberle et al. [2] have investigated electron-beam CT for reconstructing images of two-phase flows, and Schleicher et al. [3] have introduced the design concept of an optical tomograph.

Several researchers have reported different types of flow pattern detection schemes. For example, Mi et al. [4] used a neural network for identification of two-phase flow patterns in a vertical channel using signals from electrical capacitance probes. Warsito and Fan [5] used a neural network-based multicriteria optimization image reconstruction technique for imaging multiphase flow systems by electrical capacitance tomography. Gao and Jin [6] proposed a flow pattern complex network (FPCN) based on conductivity data. However, all these techniques suffer from the disadvantage of using an invasive sensor.

Ultrasonic sensing offers an alternative method that has many benefits compared to other available methods. For example, in addition to its noninvasive nature, an ultrasonic measurement system is portable, independent of its host, and can be conveniently implemented. However, a majority of the current ultrasonic methods are limited by the fact that they also rely on prior signal attenuation calibration.

Chakraborty et al. [7] proposed a noninvasive ultrasonic method for flow pattern classification, where a one-to-one correspondence was established between the flow measure obtained from symbolic analysis of ultrasonic data and the void fraction measured from a conductivity probe. The work reported in this paper is an extension of the earlier work [7]. From this perspective, major contributions and specific features of this paper are delineated below.

- Usage of ultrasonic wave reflections from the bubbles: This is a significant improvement over the earlier work [7] that used reflections from the wall of a flow channel. Wall reflections might not be very useful in practice, because they are attenuated to a large extent and thus lose significant information of the flow characteristics.
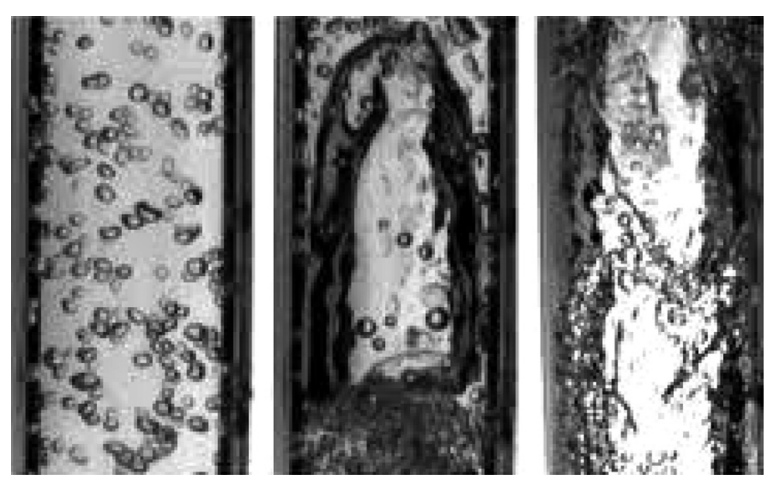

Fig. 1 Illustration of three different patterns of two-phase gas-liquid flow. From left to right: bubbly, cap-bubbly, and slug flow patterns. 
- Classification of multiple types of two-phase flow patterns: While earlier work [7] established a one-to-one correspondence between the void fraction and a flow measure by symbolic analysis, it did not explicitly perform classification of two-phase flow patterns. The classification algorithm, reported in this paper, identifies the flow pattern with good accuracy. Apparently, such a classification scheme using a single ultrasonic sensor has not yet been reported in open literature.

- Computational speed of the classification algorithm: The average execution time of the algorithm for a flow pattern classification on a typical onboard platform is $\sim \frac{1}{8} \mathrm{~s}$, which is much less than the time interval between two consecutive testings for identification of the flow regime in industrial applications.

\section{Review of Underlying Concepts}

The classification scheme presented in this paper is built upon partitioning of the observed time series to obtain a symbol block that, in turn, is used to construct probabilistic finite state automata (PFSA) [8,9]. In this paper, a suboptimal partitioning scheme has been adopted [10] after selecting an appropriate alphabet size [11]. While the details of these methods are reported in the respective afore-cited references, a brief outline of the underlying concept of symbolic dynamic filtering (SDF) [8] is presented for completeness of the paper.

(1) Data acquisition: Time-series data, denoted as $\mathbf{q}$, are acquired by sampling of sensor data from a physical process such that the data set lies within a prespecified range. Let the space of time-series data be represented as $\mathcal{Q} \subseteq \mathbb{R}^{L}$, where the positive integer $L$ is the length of the collected data and $L$ is chosen to be sufficiently large for convergence of statistical properties within a specified threshold.

(2) Construction of symbol blocks: The time series is symbolized by introducing a partition $\mathbb{B} \triangleq\left\{B_{0}, \ldots, B(|\Sigma|-1)\right\}$ that consists of $|\Sigma|$ mutually exclusive, and exhaustive cells over the range of the data set, where each cell is labeled by a symbol $\sigma_{j} \in \Sigma$ and $\Sigma=\left\{\sigma_{0}, \ldots, \sigma_{|\Sigma|-1}\right\}$ is called the alphabet of symbols. This step enables transformation of the time series $\mathbf{q}$ into a symbol block $\mathbf{s}$ of length $L$, consisting of the symbols in the alphabet $\Sigma$, and is analogous to coarse-graining or quantization.

(3) Construction of a PFSA: First, a PFSA structure is constructed, which is an $r$-state Markov machine. Then, a symbol block $\{\mathbf{s}\}$ of length $L$ is run through the PFSA to generate an irreducible $(r \times r)$ state transition matrix $\Pi \equiv\left[\pi_{j k}\right]$, where $\pi_{j k} \geq 0$ is the transition probability from state $j$ to state $k$ of the PFSA. The resulting state probability vector $\mathbf{p}=\left[p_{1} \cdots p_{r}\right]$ is the left eigenvector corresponding to the unique unity eigenvalue of $\Pi$ that could be treated as the extracted feature vector.

2.1 Optimization of Partitioning. The process of partitioning is crucial for pattern classification of the time series. The primary objective here is to effectively capture the change in information contents in the time series belonging to different classes. Sarkar et al. [10] have introduced a time-series partitioning scheme, called the wrapper method, that aims to minimize the pattern classification error for statistically unstructured applications. In view of the (possibly) non-Gaussian distribution of the two-phase flow problem, the wrapper method is adopted in this paper.

A composite cost based on a confusion matrix $\mathbf{C}$ is minimized for multi-objective optimization, where each element $c_{i j}$ of $\mathbf{C}$ denotes the frequency of data from the class $\mathrm{Cl}_{i}$ being identified as data from the class $C l_{j}$ [12]. The composite cost consists of two costs that are defined by using an additional penalty weighting matrix, elements of which represent the relative penalty for different confusions in the classification process.

Let there be $n$ classes of data labeled $C l_{1}, \ldots, C l_{n}$, and let $\mathbf{W}$ be the weighting matrix, where each of its elements $w_{i j}$ denotes the penalty incurred by classifying data from class $C l_{i}$ as data from class $C l_{j}$. With these definitions, the expected cost $\left(\operatorname{Cost}_{E}\right)$ due to classification errors is defined as

$$
\operatorname{Cost}_{E} \triangleq \frac{1}{N_{s}}\left(\sum_{i} \sum_{j} w_{i j} c_{i j}\right)
$$

where $N_{s}$ is the total number of training samples for all classes. We also minimize the cost, Cost $_{W}$, of worst case classification error, which depends on the partitioning $\mathbb{B}$.

The cost $\operatorname{Cost}_{W}$ is defined as

$$
\operatorname{Cost}_{W} \triangleq \max _{i}\left(\frac{1}{N_{i}} \sum_{j} w_{i j} c_{i j}\right)
$$

where $N_{i}$ is the number of training samples in the class $C l_{i}$. A partitioning $\mathbb{B}$ generates the feature of each sample for pattern classification, where the k-NN classifier has been adopted because of its computational advantages [13].

A composite error cost $\operatorname{Cost}_{O}$ is constructed for multi-objective optimization by a convex combination of $\operatorname{Cost}_{E}$ and Cost $_{W}$ as

$$
\operatorname{Cost}_{O} \stackrel{\Delta}{=} \alpha \operatorname{Cost}_{E}+(1-\alpha) \operatorname{Cost}_{W}
$$

where the scalar parameter $\alpha \in[0,1]$ assigns relative importance to the individual costs.

2.1 Optimization Procedure. The objective of the partitioning scheme is to obtain a (sub)optimal partitioning $\mathbb{B}^{*}$ that

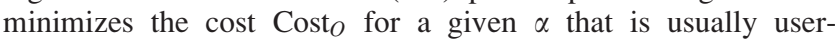
specified

$$
\mathbb{B}^{*}=\arg \min _{\mathbb{B}} \operatorname{Cost}_{O}(\mathbb{B})
$$

The above optimization procedure could be repeated for a range of $\alpha$ to construct a Pareto front [14].

The compact range of the time series is determined from the maximum and minimum values of the observed data and a sufficiently fine set of grid points is overlaid on this range. Let the number of grid points be $G$. Each of the grid points denotes a possible position of a partitioning cell boundary. In other words, a partitioning that has $|\Sigma|$ symbols would require $(|\Sigma|-1)$ partitioning boundaries that could be selected from $G$ grid points. As a notation, a $\Sigma$-cell partitioning $\mathbb{B}$ is expressed as $\Lambda_{|\Sigma|} \triangleq\left\{\lambda_{1}, \lambda_{2}\right.$, $\left.\ldots, \lambda_{|\Sigma|-1}\right\}$, where $\lambda_{i}$ denotes a partitioning boundary. Since the number of possible partitionings grows rapidly with an increase in $G$ and $|\Sigma|$, a sequential search based algorithm [10] is adopted to obtain a (sub)optimal solution. The underlying procedure is described as follows:

- The first step is to search for the optimal grid point that divides the range of the data into two cells. $\Lambda_{2}=\left\{\lambda_{1}\right\}$, where $\lambda_{1}$ is evaluated as

$$
\lambda_{1}^{*}=\arg \min _{\lambda_{1}} \operatorname{Cost}_{O}\left(\Lambda_{2}\right)
$$

where the two-cell optimal partitioning is given by $\Lambda_{2}^{*}=\left\{\lambda_{1}^{*}\right\}$.

- The next step is to partition the range of the data into three cells by dividing either of the two existing cells of $\Lambda_{2}^{*}$ with 
the placement of a new partition boundary at $\lambda_{2}$. $\lambda_{2}$ is given by

$$
\lambda_{2}^{*}=\arg \min _{\lambda_{2}} \operatorname{Cost}_{O}\left(\Lambda_{3}\right)
$$

where the three cell partition $\Lambda_{3}=\left\{\lambda_{1}^{*}, \lambda_{2}\right\}$. The (sub)optimal three-cell partitioning is obtained as $\Lambda_{3}^{*}=\left\{\lambda_{1}^{*}, \lambda_{2}^{*}\right\}$. In this (local) optimization procedure, the cell that provides the largest decrement in $\mathrm{Cost}_{O}$ upon further segmentation ends up being partitioned.

- Iteratively, this procedure is extended to obtain the $m$-cell partitioning as follows:

$$
\lambda_{|\Sigma|-1}^{*}=\arg \min _{\lambda_{|\Sigma|-1}} \operatorname{Cost}_{O}\left(\Lambda_{|\Sigma|}\right)
$$

where $\Lambda_{|\Sigma|}=\Lambda_{|\Sigma|-1}^{*} \cup\left\{\lambda_{|\Sigma|-1}\right\}$ and the optimal $|\Sigma|$ cell partitioning is given by $\Lambda_{|\Sigma|}^{*}=\Lambda_{|\Sigma|-1}^{*} \cup\left\{\lambda_{|\Sigma|-1}^{*}\right\}$.

- The optimization procedure is monotonically decreasing [10] in the cost function with every additional sequential operation, i.e., $\operatorname{Cost}_{O}\left(\Lambda_{|\Sigma|-1}^{*}\right) \geq \operatorname{Cost}_{O}\left(\Lambda_{|\Sigma|}^{*}\right)$. The process of creating additional partitioning cells is stopped if the cost decrease falls below a specified positive scalar threshold $\eta_{\text {stop }}$ and the stopping rule is: $\Lambda_{|\Sigma|-1}^{*}$ is the optimal partitioning if

$$
\left(\operatorname{Cost}_{O}\left(\Lambda_{|\Sigma|-1}^{*}\right)-\operatorname{Cost}_{O}\left(\Lambda_{|\Sigma|}^{*}\right)\right) \leq \eta_{\text {stop }}
$$

where the user-defined scalar parameter $\eta_{\text {stop }}$ is chosen for making trade-offs between computational load and error reduction (e.g., loss of information). While a large $\eta_{\text {stop }}$ may create a coarse partitioning of a data set leading to unacceptable loss of information, a fine partitioning due to a small $\eta_{\text {stop }}$ may decrease robustness to spurious noise and increase the computational load without any significant improvement in the results [11]. In essence, the choice of an appropriate

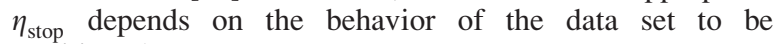
partitioned.

\section{Experimental Validation on a Test Apparatus}

This section experimentally validates the concept of pattern classification in a two-phase flow process by ultrasonic sensing in a laboratory environment. Different components of the test apparatus are briefly described below:

- Ultrasonic probe: The ultrasonic transducer is placed on an acrylic block which conforms to the shape of the pipe in the test section. A Matec PR5000 sine wave pulser/receiver is used to inject a pulsed sine wave ultrasonic wave into the pipe. The signal is captured at the rate of 100 megasamples per second by a National Instruments PCI-5122 oscilloscope card installed on a desktop computer. The oscilloscope is triggered using the digital trigger pulse generated by the pulser/receiver. An ultrasonic transducer $(3.5 \mathrm{MHz}, 12.5 \mathrm{~mm}$ diameter Panametrics A415S) is placed on the acrylic block of thickness $13.5 \mathrm{~mm}$ that conforms to the outside diameter of the acrylic pipe; this makes a total thickness of $20 \mathrm{~mm}$ when added to a pipe wall of $6.5 \mathrm{~mm}$ thickness. The pulser/ receiver is used in the pulse-echo mode to produce a $3.5 \mathrm{MHz}$ pulsed sine wave of $\sim 5.0 \mu$ s during a repetition rate of 400 pulses per second. Only a portion of the resulting signal is used for analysis. There are two types of echoes that one should expect in the present settings.

(1) The reflections from the bubbles in the flow.

(2) The back wall reflections, i.e., the reflections from the wall of the pipe.
The reflections from the back wall are attenuated to a great extent and, in some cases, contain no information at all. However, the reflections from the bubbles exist for a small period of time and hence may not be able to capture the characteristics of the flow. Reflections of individual ultrasonic pulses contain information corresponding to a time-localized snap-shot of the two-phase flow. However, relevant statistical information, which is a representative of time-averaged flow condition, can only be extracted by compiling a large ensemble of pulses. Working with such a data set ensures that the effects of spatial distribution of voids are incorporated in the analysis. Typical wave forms of ultrasonic refections are displayed in Figs. 2(a)-2(c) for bubbly flow, cap-bubbly flow, and slug flow, respectively. These figures show the temporal variations of reflections and justify the fact that a single pulse may not be able to capture the true characteristics of the flow pattern. Therefore, it is necessary to stack several of these reflections together for capturing the actual dynamics of flow. Upon compilation of 50 such reflections at the rate of $\sim 400$ per second, i.e., $\sim \frac{1}{8} \mathrm{~s}$ for 50 reflections, a single data point is generated for pattern classification.

\section{Results and Discussion}

This section presents the following experimental results that were generated by analyzing the ultrasonic data:

(1) 1060 data points for bubbly flow.

(2) 485 data points for cap-bubbly flow.

(3) 260 data points for the slug flow.

From each class 250 data points were divided equally into training and test data sets. The data points were shuffled randomly 100 times so as to obtain the statistical behavior of error in the classification scheme. As discussed earlier, for partitioning the data, a weighting matrix $\mathbf{W}$ needs to be defined to calculate the costs $\operatorname{Cost}_{E}$ and $\operatorname{Cost}_{W}$ from the confusion matrix for the training data set. The weighting matrix $\mathbf{W}$ is chosen as shown in Table 1.

There is no penalty for correct classification, i.e., each diagonal element of the weighting matrix $\mathbf{W}$ is zero. For the off-diagonal elements, a weight $w_{i j}$ represents the penalty assigned for incorrectly identifying a class $C l_{i}$ data as a $C l_{j}$, where $i \neq j$. In this problem, an incorrect classification of a flow pattern, having a larger departure from the single-phase liquid, as a flow pattern, having a smaller departure from the single-phase liquid, is considered to be more risky than the corresponding reverse decision. For example, the penalty for classifying a slug flow as a bubbly flow is larger than that for classifying a bubbly flow as a slug flow. That is, the weighting matrix $\mathbf{W}$ has to follow two rules: (i) $w_{i i}=0$ and (ii) $w_{i j}>w_{j i}$ if $i>j \forall i, j \in\{1,2,3\}$.

The data space region $\Omega$ is divided into 40 grid cells, i.e., 39 grid boundaries excluding the boundaries of $\Omega$. The sequential partitioning optimization procedure described in Sec. 2.1 is then employed to identify the optimal partitioning.

The parameter $\alpha$ in Eq. (3) is set to 0.5 to put equal weights on $\operatorname{Cost}_{E}$ and $\operatorname{Cost}_{W}$ in construction of the composite cost Cost $O$. The threshold $\eta_{\text {stop }}$ in Eq. (8) is chosen to be $10^{-3}$, i.e., the suboptimal search for partitioning the data is recursively continued until the error in Eq. (8) is less than $10^{-3}$. For this case, the partitioning creates seven cells, i.e., the alphabet size becomes $|\Sigma|=7$ based on an entropy-rate criterion [11]. For symbolic dynamic analysis, the depth of the D-Markov machine for PFSA construction [8] is taken to be $D=1$ because a higher value (i.e., $D \geq 2$ ) does not yield any significant increase in (entropy-rate-based) performance in this specific case. The features are classified by a k-NN classifier (with $k=5$ ) using the Euclidean distance metric [13].

The probability distribution of the classification error was generated by running the algorithm for 100 times. Using the $\chi^{2}$ goodness of fit, the error statistics were approximated as a Gaussian distribution with a significance level of $10 \%$. The mean error is $\sim 11.40 \%$ and the standard deviation is $\sim 2.20 \%$, which is 


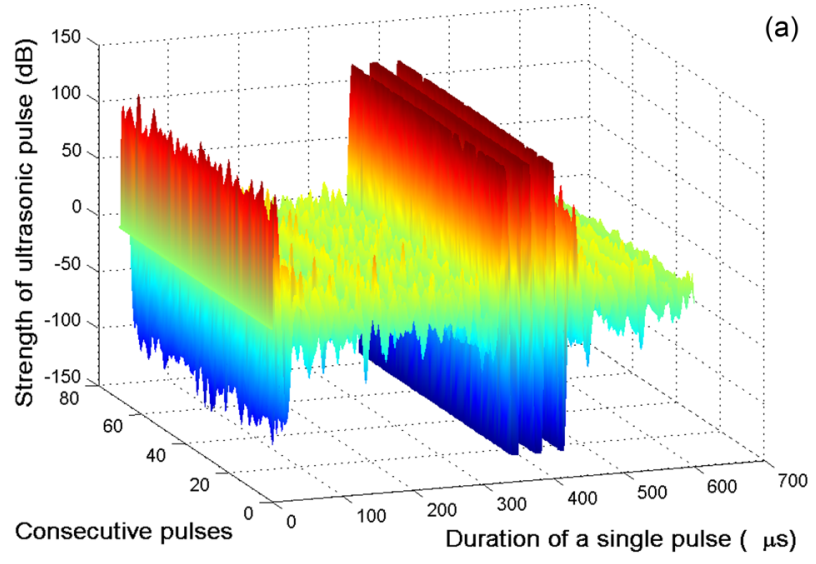

(b)

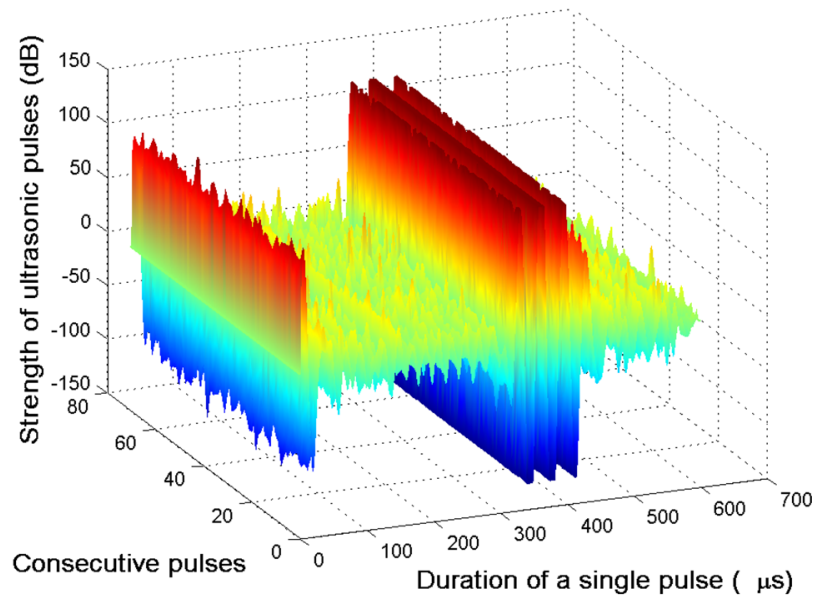

(c)

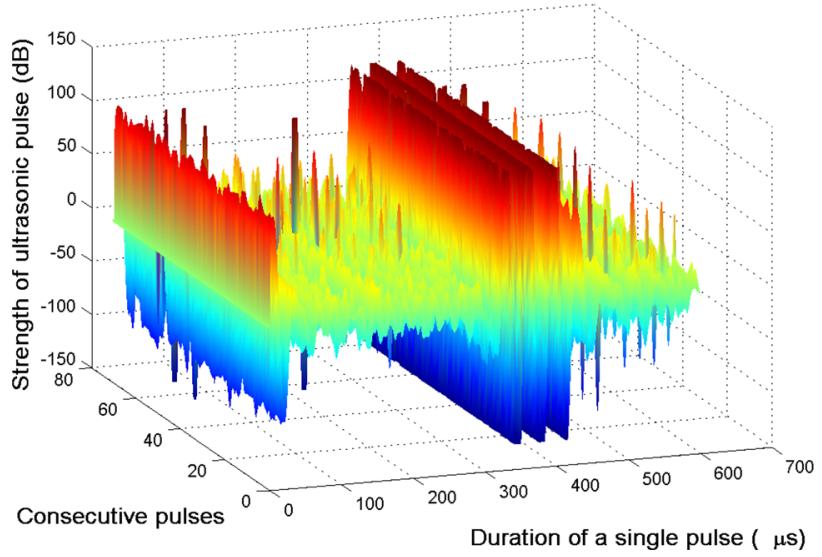

Fig. 2 Ultasonic reflections for bubbly, cap-bubbly, and slug flow. The $x$-axis in each plot represents the time corresponding to "duration of a single pulse," while the $y$-axis and $z$-axis represent "consecutive pulses" and "strength of ultrasonic pulses," respectively.

Table 1 Partitioning parameters: weighting matrix W

\begin{tabular}{lccc}
\hline \hline Ground truth versus classification results & Bubbly & Cap-bubbly & Slug \\
\hline Bubbly & 0 & 2 & 3 \\
Cap-bubbly & 4 & 0 & 2 \\
Slug & 8 & 4 & 0 \\
\hline \hline
\end{tabular}

Table 2 Classification results: confusion matrix C

\begin{tabular}{lrrr}
\hline \hline Ground truth versus classification results & Bubbly & Cap-bubbly & Slug \\
\hline Bubbly & 103 & 17 & 5 \\
Cap-bubbly & 12 & 105 & 8 \\
Slug & 3 & 5 & 117 \\
\hline \hline
\end{tabular}

considered to be a satisfactory performance [15]. Apart from diminishing the error rates, the goal is to reduce the classification errors (e.g., identifying a slug flow as a bubbly flow). As an example, a typical confusion matrix $\mathbf{C}$ is obtained by execution of the pattern classification algorithm as shown in Table 2, where the rows indicate the truth of pattern identification in the order of bubbly, cap-bubbly, and slug flows from left to right, and the columns indicate the results of pattern identification in the order of bubbly, cap-bubbly, and slug flows from top to bottom.

For the current choice of the weighting matrix $\mathbf{W}$, an examination of the above confusion matrix $\mathbf{C}$ reveals that the chances of correct decision are very high compared to those of incorrect decisions. Furthermore, the chances of slug flow samples being classified as bubbly or cap-bubbly flow are relatively low compared to the reverse decisions. Similarly, the chances of capbubbly flow being classified as bubbly flow are lower than the reverse decision. The results of pattern classification, obtained as the confusion matrix $\mathbf{C}$, are strongly dependent on the choice of the weighting matrix $\mathbf{W}$ that puts more penalty on misclassifying a flow pattern (e.g., slug) as a less severe flow pattern (e.g., bubbly) than that for the reverse decision. The results are in agreement with what is expected, because the partitioning costs are minimized if the number of severe misclassifications is fewer than that of benign misclassifications.

\section{Conclusions and Future Work}

This paper develops and experimentally validates the concept of a method for classification of two-phase flow patterns using noninvasive measurements from a single ultrasonic sensor. The proposed method allows for online classification of two-phase flow patterns using relatively inexpensive hardware at a low computational cost. These advantages are particularly relevant for both local and remote sensing applications.

This method of flow pattern classification and the associated flow regime identification is applicable to a wide range of flow channels at high void fractions and can be implemented in a variety of industry (e.g., petrochemical processes and nuclear power plants). From these perspectives, future research is recommended in the following areas.

(1) Multisensor information fusion: While multisensor ultrasonic techniques would provide a more complete picture of the flow field, extensive data processing is required to reduce the ensemble of data for classification of a flow pattern. Future research should focus on efficient methods for capturing codependence among different sensors for information fusion.

(2) Testing with different channel geometry: Additional testing should be conducted with larger flow channel sizes with different shapes and orientation to allow for variations in flow patterns.

\section{Acknowledgment}

The authors are thankful to Mr. Mohan Yadav and Dr. Seungin Kim for providing the experimental data, and to Dr. Eric Keller for assistance in the interpretation of the experimental data. This work has been supported in part by the U.S. Army Research Laboratory and the U.S. Army Research Office under Grant No. 
W911NF-07-1-0376. Any opinions, findings, and conclusions or recommendations expressed in this publication are those of the authors and do not necessarily reflect the views of the sponsoring agencies.

\section{References}

[1] Hewitt, G., Delhaye, J., and Zuber, N., 1986, Multiphase Science and Technology, Vol. 2, Springer, New York, NY.

[2] Bieberle, M., Schleicher, E., and Hampel, U., 2008, "Simulation Study on Electron Beam X-Ray CT Arrangements for Two-Phase Flow Measurements," Meas. Sci. Technol., 19, p. 094003.

[3] Schleicher, E., Silva, M. J., Thiele, S., Li, A., Wollrab, E., and Hampel, U., 2008, "Design of an Optical Tomograph for the Investigation of Single- and Two-Phase Pipe Flows," Meas. Sci. Technol., 19, p. 094006.

[4] Mi, Y., Ishii, M., and Tsoukalas, B., 2001, "Flow Regime Identification Methodology With Neural Networks and Two-Phase Flow Models," Nucl. Eng. Des., 204, pp. 87-100.

[5] Warsito, W., and Fan, L.-S., 2001, "Neural Network Based Multi-Criterion Optimization Image Reconstruction Technique for Imaging Two- and ThreePhase Flow Systems Using Electrical Capacitance Tomography," Meas. Sci. Technol., 12, pp. 2198-2210.
[6] Gao, Z., and Jin, N., 2009, "Flow-Pattern Identification and Nonlinear Dynamics of Gas-Liquid Two-Phase Flow in Complex Networks," Phys. Rev. E: Nonlin. Plasma Phys., Fluid, Dyn., Rel. Topics, 79(Pt 2), p. 066303.

[7] Chakraborty, S., Keller, E., Talley, J., Srivastav, A., Ray, A., and Kim, S. 2009, "Void Fraction Measurement in Two-Phase Processes via Symbolic Dynamic Filtering of Ultrasonic Signals," Meas. Sci. Technol., 20, p. 023001.

[8] Ray, A., 2004, "Symbolic Dynamic Analysis of Complex Systems for Anomaly Detection," Signal Process., 84, pp. 1115-1130.

[9] Vidal, E., Thollard, F., de la Higuera, C., Casacuberta, F., and Carrasco, R. 2005, "Probabilistic Finite-State Machines-Part I and Part II," IEEE Trans. Pattern Anal. Mach. Intell., 27(7), pp. 1013-1039.

[10] Sarkar, S., Mukherjee, K., Jin, X., Singh, D., and Ray, A., 2012, "Optimization of Symbolic Feature Extraction for Pattern Classification," Signal Process., 92(3), pp. 625-635.

[11] Rajagopalan, V., and Ray, A., 2006, "Symbolic Time Series Analysis via Wavelet-Based Partitioning," Signal Process., 86(11), pp. 3309-3320.

[12] Freitas, A., 2002, Data Mining and Knowledge Discovery With Evolutionary Algorithms, Springer-Verlag, Berlin.

[13] Bishop, C., 2006, Pattern Recognition and Machine Learning, Springer, New York, NY.

[14] Miettinen, K., 1998, Nonlinear Multiobjective Optimization, Kluwer International, Boston, MA.

[15] Brunk, H., 1975, An Introduction to Mathematical Statistics, Xerox College Publishing, Lexington, MA. 